\title{
VELOCITY DETECTION FROM A MOTION BLUR IMAGE USING RADON TRANSFORMATION
}

\author{
Tantra Nath Jha*
}

\begin{abstract}
Motion blur is the result when the camera shutter remains open for an extended period of time and a relative motion between camera and object occurs. An approach for velocity detection based on motion blurred images has been implemented by the Radon transformation. The motion blur parameters are first estimated from the acquired images by using Radon transformation and then used to detect the speed of the moving object in the scene. Here established a link between the motion blur information of a 2D image and camera manufacturer's data sheet and its calibration
\end{abstract}

Keywords: Blur, shutter, transformation, motion, camera.

\section{INTRODUCTION}

A picture is a set of pixels, each contain an average value of light caught in time of exposure the time frame. When the subject does not move during the time frame (and is in focus), it will appear frozen in the image and we will get sharper edges. Blur occur when a cell (or pixel) catch light from more than one point at the scene (Mohsen Ebrahimi Moghaddam, Mansour Jamzad,, 2007). Blur is very common in pictures. When taking picture with blur, a lot of information lost. The motion blur parameters are first estimated from the acquired images and then used to detect the velocity of the moving object in the scene (Huei-Yung Lin and Kun-Jhih Li, 2004). Here established a link between the motion blur information of a 2D image and the velocity information of a moving object.

\section{BACKGROUND THEORY}

The blur problem is encountered in many different technical areas, such as Astronomical imaging, remote sensing, microscopy, medical imaging, optics, photography, super resolution applications, and motion tracking applications among others. Extraterrestrial observations of the Earth and the planets are degraded by motion blur as a result of slow camera shutter speeds relative to the rapid space craft motion

* Mr. Jha is associated with Electronics and Computer Department, T.U. I.O.E, Purwanchal campus, Dharan. 
Hence velocity of the vehicle (moving object) can be found by a single snap photograph.

The image restoration is to reconstruct or estimate the uncorrupted image by using the degraded version of the same image. Generally, an image can be corrupted by motion blur and additive noise. Eq. (1) shows the relation between the observed image $g(x, y)$ and its uncorrupted version $f(x, y)$

$$
g(x, y)=f(x, y) * h(x, y)+n(x, y),---E q . ~(1)
$$

Where $\mathrm{h}$ is the blurring function or point spread function (PSF) that convolves an original image and $n$ is the additive noise function. Image restoration algorithms suppose that only the observed image is available and no extra information about other parameters exists. According to Eq. (1), in order to determine the uncorrupted image, we need to find the blurring function $h$. Therefore, one of the major problems is to find an exact estimation of $h$

\section{Motion blur attributes}

The general form of motion blur function is given as (Mohsen Ebrahimi Moghaddam, Mansour Jamzad, 2007)

$$
h(x, y)=\frac{1}{L} \text { if } \sqrt{x^{2}+y^{2}}<\frac{L}{2} \text { and } \frac{x}{y}=-\tan (\vartheta)------\quad \quad \text { Eq-2 }
$$

\section{Motion blur parameter estimation}

Here propose a solution for cases in which the image is corrupted only by a degradation function without additive noise (i.e. $n(x, y)=0$ ). In the absence of noise, Eq. (1) implies that $\mathrm{G}(\mathrm{u}, \mathrm{v})=\mathrm{F}(\mathrm{u}, \mathrm{v}) \mathrm{H}(\mathrm{u}, \mathrm{v})$, where $\mathrm{G}(\mathrm{u}, \mathrm{v}), \mathrm{F}(\mathrm{u}, \mathrm{v})$ and $\mathrm{H}(\mathrm{u}, \mathrm{v})$ are frequency response of observed image, original image and degradation function, respectively. In this case, the motion blur parameters are determined as explained in the system below.

\section{Radon Transformation}

According ((Høilund, November 12, 2007) Radon Transform on an image $f(x, y)$ for given set of angles can be thought of as computing the projection of the image along the given angles. The resulting projection is the sum of the intensities of the pixels in each direction, i.e. a line integral. The result is a new image $R(\rho, \alpha)$. This is depicted in Figure on the facing page. 
This can be written mathematically by defining

$\mathrm{r}=x \cos \alpha+y \sin \alpha \quad---$ Eq. (3).

After which the Radon transform can be written as $R(\rho, \theta)=\iint f(x, y)$ $\mathrm{d}(\mathrm{r}-\mathrm{x} \cos \alpha-\mathrm{y} \sin \alpha) \mathrm{dx} \mathrm{dy}$.

----Eq. (4)

The complete Radon transform of the image. The white spot is the distance from the center and the

Angle at which the sum of intensities in the image peaks. It is thus the slope of the line along with the position.

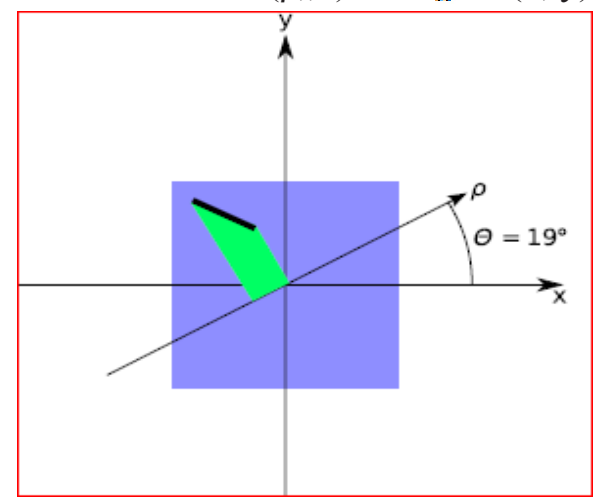

\section{Motion direction estimation}

To find the motion direction, we used the parallel dark lines that appear in frequency response of degraded image as shown in Fig. It can be known the motion blur direction $(\Phi)$ is equal to the angle $(\varnothing)$ between any of these parallel dark lines and the vertical axis .To find the motion direction, Apply Radon transform on Image. Since there are parallel Dark lines in Image, high spots in $R$ will be held along a vertical line. The coordinate of this vertical line shows the direction of parallel dark lines in Image that is the motion direction $(\Phi)$.As in (Mohsen Ebrahimi Moghaddam, Mansour Jamzad, 2007 and )

\section{Motion length estimation}

After finding the motion direction as described above, it can suppose that motion is in horizontal direction, because we can rotate the axis of observed image. In this case uniform motion blur equation is given by

$$
h(i)=\left\{\begin{array}{c}
\frac{1}{L} \text { if } \frac{-L}{2} \leq i \leq \frac{L}{2}-------------E q-5 \\
0 \text { otherwise }
\end{array}\right.
$$

The continuous Fourier transform of $\mathrm{h}$ is as follow

$$
H(u)=\frac{2 \sin u L \pi / 2}{u L \pi}, 0 \leq u \leq N-1 H(u)=\frac{2 \sin u L \pi / 2}{u L \pi}
$$


Which is a SINC function, the discrete version of $\mathrm{h}$ in horizontal direction is given by (Tong, Li, Zhang, \& Zhang, 2004 and (Mohsen Ebrahimi Moghaddam, Mansour Jamzad,, 2007).

Where $\mathrm{N}$ is image dimension. $\mathrm{H}(\mathrm{u})$ is equivalence of $\mathrm{Ha}(\mathrm{u})$ in Discrete domain (we can call it a periodic SINC). To find L, we try to solve $\mathrm{H}(\mathrm{u})=0$. Solving this equation leads to solving equation $\frac{\sin u L \pi}{N}=0$ then, we can find that

$u=\frac{k^{\prime} N}{L}$ such that $k^{\prime}>0$. If we suppose that $\mathrm{u} 0$ and $\mathrm{u} 1$ are two successive zero points such that $\mathrm{H}(\mathrm{u} 0)=\mathrm{H}(\mathrm{u} 1)=0$, then we can write $u_{0}-u_{1}=N / L$ then we have

$$
L=\frac{N}{d}
$$

Where $\mathrm{d}$ is the distance between two successive dark lines in $\mathrm{G}(\mathrm{u}, \mathrm{v})$ and $\mathrm{N}$ is the size of

Motion blurred image with $\mathrm{L}=25$ pixel, $\Phi=10$

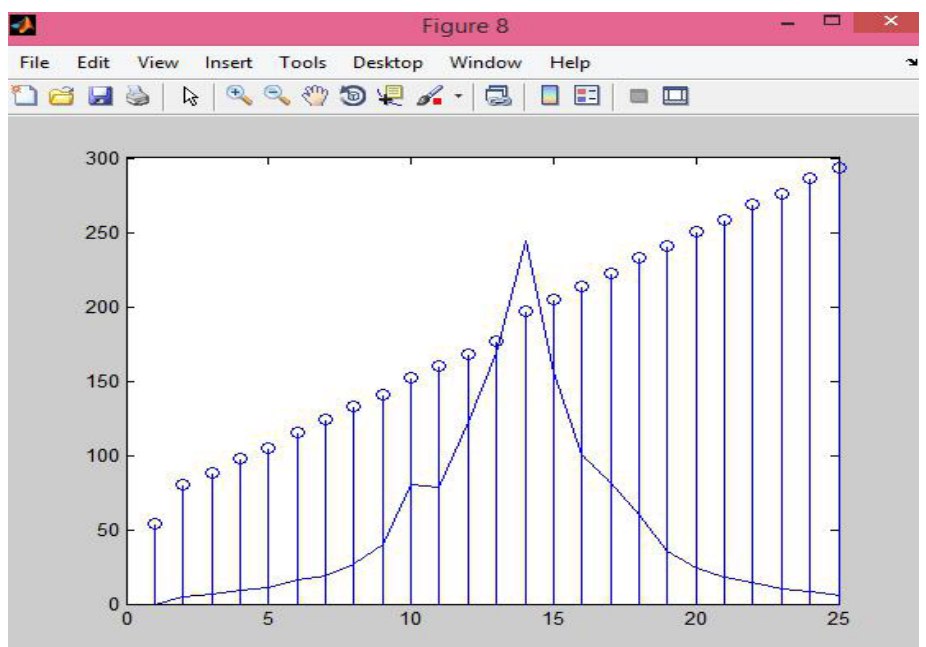

Finding local minima of above power spectrum by using moving average

\section{Velocity Detection}

Once the "length" of the motion blur identified as above, a pinhole camera model will be used to estimate the speed of the moving object. As 
shown in Figure the relationship between the distance of the object motion $d$ (in pixel) and the number of blurred pixels $K$ during a period of time is given by

2004) $\frac{d}{K}=\frac{z}{f}---------------E q .4$ (Huei-Yung Lin and Kun-Jhih Li,

Where $z$ is the distance from the camera to the moving object and $f$ is the focal length of the camera. If the shutter speed of the camera is $T$ seconds and the pixel size of the CCD in the horizontal direction is $s_{x}$ then the speed $v$ of the moving object is given by eq-5.

$$
v=\frac{s x d}{T}=z \frac{K s x}{T f}
$$

Kun-Jhih Li, 2004)

In the above equation, $\mathrm{s}_{\mathrm{x}}$ and $f$ can be obtained from the manufacturer's data sheet and camera calibration, $T$ is given by the camera setting, and $z$ is a fixed distance (between the target object and the camera) which could be measured physically (K.-J. L. C.-H. C. Huei-Yung Lin*, "Vehicle speed detection from a single motion blurred image, 2008)

\section{RESULTS}

The comparative result of 3 degraded image has been analysed as follows:

\section{Comparison of Result on 3 Degraded Image}

\begin{tabular}{|l|l|l|l|}
\hline Image & $\begin{array}{c}\text { Convoluted } \\
\text { Length, } \\
\text { Angle }\end{array}$ & $\begin{array}{c}\text { Calculated Length } \\
\text { in } \\
\text { Pixel and Angle in } \\
\text { Degree }\end{array}$ & \multicolumn{1}{|c|}{$\begin{array}{c}\text { Error in } \\
\text { Percentage (\%) }\end{array}$} \\
\hline \multirow{4}{*}{ a)Lina } & i) 30,15 & i) $31.4410,16$ & i) $4 ., 6.66$ \\
\cline { 2 - 4 } & ii) 30,45 & ii) $27.5180,47$ & ii) $8.27,4.44$ \\
\cline { 2 - 4 } & iii) 30,80 & iii) 24,81 & iii) $20,1.25$ \\
\hline \multirow{2}{*}{ b)Cameramen } & i) 30,10 & i) $30.2510,11$ & i) $0.8366,10$ \\
\cline { 2 - 4 } & ii) 30,80 & ii) $28.6135,81$ & ii) $4.5883,1.25$ \\
\hline \multirow{2}{*}{ c) Toy car } & i) 20,30 & i) $24.1327,38$ & i) $20.66,26.66$ \\
\cline { 2 - 4 } & ii) 30,15 & iii) $32.0481,20$ & ii) $6.827,33.333$ \\
\hline
\end{tabular}


Experimental result of our algorithm on 3 degraded standard images with No additive noise using noise free method by radon transformation using Mat-lab

Given following parameters: focal length $\mathrm{f}=12 \mathrm{~mm}, \mathrm{CCD}$ pixel size $\mathrm{sx}=0.011 \mathrm{~mm}$, shutter speed $\mathrm{T}=1 / 100 \mathrm{~s}$ and Distance to the object $\mathrm{z}=575 \mathrm{~mm}$. The length 25 pixels. The speed of the moving object was calculated using Eq...(10) The estimate of the speed was found to be 1329 $\mathrm{mm} / \mathrm{s}$ (K.-J. L. C.-H. C. Huei-Yung Lin*, "Vehicle speed detection from a single motion blurred image, 2008)

Actually calculated blur length 25.974 pixel on self-blind convoluted image of and length 25.9714 pixel on unknown blur image respectively, so

Velocity $=1368.9 \mathrm{~mm} / \mathrm{sec}$ and $1368.9 \mathrm{~mm} / \mathrm{sec}$ as Eq. ... (10)

Error in velocity measure (1329-1368.9)/1329=3.0022 percent.

\section{CONCLUSION}

The Radon Transform is used for extracting motion blur parameter from a single image taken by CCD camera. Our method improved the accuracy of speed estimation and measurement of motion blur parameters. In this case, a camera model with objects moving non-parallel to the image plane has been considered

\section{REFERENCES}

Høilund, C. (2007). The Radon Transform. Aalborg University, VGIS, $07 g r 721$.

Huei-Yung Lin and Kun-Jhih Li. (2004). Speed detection. Image Processing, ICIP.

K.-J. L. C.-H. C. Huei-Yung Lin*, Vehicle speed detection from a single motion blurred image. Image and Vision Computing. 26 2008).

Mohsen Ebrahimi Moghaddam, Mansour Jamzad,. (2007). [MoMotion blur identification in noisy images using mathematical models and statistical measures. Elsevier Ltd.

Tong, H., Li, M., Zhang, H., Zhang, C. (2004). Blur detection for digital images using wavelet transform. IEEE international conference on Multimedia, 1, 17-20.

Z. Devcic, S. Loncaric. (2000). Blur identification using average spectra. IEEE International Conference on Acoustics, Speech, and Signal, 2195-2198. 